16 rofroidissement est plus lent. Il est donc nécessaire de rofroidir aussi rapidement que possible le molange gazenx. los conditions ne sont pas aisées a remplir, el est un Norvógien, M. Birkeland, qui réussit le premier, en 1003, à les róaliser industrielloment; son procédé est, depuis 1905, appliqué à Notodden.

Lo four Birkeland est conslitué nar une enceinte réfractaire, limitant une cavité cylindrique de fable épaisseur ; un violent courant d'air pénètre dans celte cavité par des ouverlures centrales et s'échappe par un canal périphérique. Les clectrodes, liges de cuivre creuses, refroidies par circulalion d'oau, sont disposées radialement et vennent aboutir dans laxo du lour, en regard des masses polaires d'un puissint électro-dimant. Sous l'action du champ magnétique intonse dévoloppé par l'électro-aimant, l'arc voltaique prend los appuncones d'un disque de flamme, qui emplit la cavité du lour. Jos fours actuels absorbent environ $1000 \mathrm{HP}$, el le disquo de llamme a plus de $2 \mathrm{~m}$. de diametre. Au cours des ossais, la puissance du four a été portée à $1500 \mathrm{HP}$, et le diamètre alleignail alor's près de 3 mètres.

Les grandes lignes do la fabrication peuvent se résumer ainsi. Cn ventilaleur puissant envoie 25 mètres cubes d'air par mimute dans le cour, au sortir duquel le mélange gazoux passe dans les refroidisseurs; de là, les gar entrent dans les tours doxydalion, où le bioxyde d'azole se transforme en peraxyde. Hs sont cnsule dirigés dans les tours d'absorption, perecuruos par un courant d'eau qui fixe, à l'état d'acide nibringe, 80 pour 100 des gaz nilreux; les 20 pour 100 qui restunt sont a peu près lotalement ahsorbés dans les deux donieres lours, renfermant du carbonate de sodium, et domnent du nitrate et du nitrite de sodium. Liacide nitrique produil est saturé par du calcaire, et transformé ainsi en nibrate de chaux solide ; de mème, on fait cristalliser le nitrite de soude provenant des deux dernières tours.

Le nitrate norvégien est livré au eommerce dans des barils en bois de $100 \mathrm{kgs}$ et de $20 \mathrm{~kg} s$, à un prix légèrement inférieur a celui du nitrate du Chili. Au point de vue agricole, laclion fertilisante des deux nitrates est la même; le produil synthétique a le léger inconvénient d'être hygroscopique, de sorte qu'il ne faut ouvrir le baril qu'au moment de lemploi. Lo nilrile de soude norvégien est très recherché dans l'industrio des matieres colorantes : Lyon on consomme la majeure partie. Fnfin, depuis 1908, Notodden fabrique de l'acide azoliquo à 100 pour 100.

Le rendement du procédé Birkeland est de $450 \mathrm{kgs}$ à 500 kigs d'acide azotique à 100 pour 100 par kilowatt-an ; le prix de revient, ne diépasserait pas $1 \mathrm{fr}, 20$.

Fn 1005, l'usine de Notodden ne disposait que de $2.500 \mathrm{HP}$ depuis 1907, elle absorbe 40.000 He qui lui sont fournis mar In station de force de Svaclgfos. L'usine renferme, depuis 1008. 36 fours do $833 \mathrm{kw}$. chacun, et produit annuellement 32.000 tonnes de nitrate de chaux.

Au reste, des travaux colossaux d'amenagement de chutes sont on cours d'exécution. Fn 1910. l'usine de Saaheim, voisino de colle de Notodden. rocevra à son tableau de distribution uno puissance de 110.000 HP, qui sera portée en 1913 ; i $227.000 \mathrm{HP}$. En 1920, la puissance totale absorbóo par los usines à nitrates norvégiennes sera de $500.000 \mathrm{HP}$. Leur production afteindra en 1911, 125.000 tonnes : en 1913. 200.000 tonnes : en 1920,300.000 tonnes. Les dénenses dé premier établissement se seront éjevées à 210 millions

Il ne faut pas croire que le procédé Birkeland soit le seul mrocédé industrial de synthèse des nitrates. LiAutrichien Pauling a mis an point un four. base sur le princine du parafoudre a cornes, mais dans lequel l'arc est souffé nar un courant d'air sous pression. Co procedé est oxploitel industricllement depuis 1907 it Innshruck : deux licences du hrovet Panling ont été acquises, l'une on Talie, lautre en France : l'usine francaise, siluó à la Rnche-de-Rame, pres de Briancon, sera mise en marche dans le courant du mois de juin.
Dailleurs, des modèles nouveaux de fours à azote surgișsent tous les jours, et il est vraisenblable que quelquesuns recevront la consécration industrielle; tel le four de Schonherr, dont trois modèles d'essai de $600 \mathrm{HP}$ fonctionneraient actuellement, à Christiansand, et dans lequel l'arc électrique se développe sous forme d'une colonne de flamme de cinc mètres de hauteur ; tels encore, le four de Guye et celui de Moscicki.

Les physico-chimistes eux-mèmes ne restent pas inactifs: ils cherchent dans leurs laboratoires à pénétrer plus avant dans le mécanisme de la réaction électrothermique, et les résultats que viennent dobtenir Haber et Kœnig peuvent avoir une profonde répercussion sur l'industrie des nitrates. En opérant sous pression rédnite et avec des courants de haute tension, ciest-à-dire en favorisant lionisation gazeuse, Haber et Konig ont atteint un rendement de 14,4 pour 100 , bien incornplet de l'état actuel des industries électrothermiques alors que le rendement de 8 pour 100 n'avait jamais été dépassé.

Dans cette esquisse rapide, j'ai dû me borner à un apergu bien incomplet de l'état actue! des industries électrothermsques. Ce serait d'ailleurs mécomaitre les progrès rapides et incessants de cette technique féconde, que de la croire limitée aux seules applications que nous avons entrevues. J'aurais voulu pouvoir vous montrer presque tous les domaines de la chimie industrielle, envahis par son activité inlassable.

G'eut été vraiment trop'; et, en m'excusant davoir retenu aussi longtemps votre attention, je m'estimerai heureux, si j'ai pu éveiller en vous un peu de l'intérêt captivant que jai ressenti à étudier ces jeunes industries, qui ont apporté à nos Alpes de nouveaux éléments de prospérité, et leur ont valu la renommée, déjà lointaine, qui s'attache aux lieux l'élection des grandes conquêtes scientifiques."

\section{STATION D'ÉTUDES DE TURBINES DE RIOUPEROUX}

Dans sa très intéressante publication, qui traite d'une manière complète et documentée la question de la Mesure du Débit dans les essais de turbines hrdrauliques, $M$. E.-F. CôtE a fait ressortir "l'utilité des essais », tant pour les constructeurs que pour les industriels exploitants de chutes $\left(^{*}\right)$; et, comme tous ceux qui ont suivi de près la construction et l'exploitation des turbines hydrauliques, M. Côte conclut en émettant le vœu de voir se créer en France, et plus particulièrement à Grenoble, des stations d'essais de turbines, comme il en existe déjà en Amérique et dans certains laboratoires d'universités étrangères.

Tous les constructeurs et industriels interessés ne peuvent que s'associer à ce veu, mais il ne faut rien moins que l'inlassable activité d'hommes tels que M. Bariblion, le dévoué directeur de l'Institut Electrotechnique de Grenoble, pour nous donner l'espoir de voir ce projet réalisé dans un avenir relativement peu éloigné.

Aussi parâit-il intéressant de signaler, dans cet ordre d'jdées, la création, non point d'une i station d'essais o proprement dite, mais d'une «station d'études », pour la recherche méthodique des meilleurs procédés de construction des turbines hydrauliques, en opérant sur des modẻles réduits.

La station d'études est installée sur un terrain appartenant aux usines de Rioupríroux, c'est-à-dire en plein domaine de la « houille blanche ». Avec une parfaite amabilité,

(*) E.F. Côté, et H. BelLet. La mesture du debil dans les essais de turbines. Gratier et Rey, éditeurs, Grenoble 1 non. 
et une intelligente compréhension des intérêts de l'industrie hydraulique, qui est la source vitale des usines de Rioupéroux, la direction de ces usines a bien voulu autoriser la maison Neyret-Brenier et Cie, de Grenoble, à brancher une prise d'eau sur une des conduites principales, et à disposer, selon ses besoins, aussi bien du terrain environnant que de l'eau nécessaire.

Description de l'installation. - La prise d'eau est faite sur une des conduites principales, en un point ou la pression est de $30 \mathrm{~m}$. environ. Un robinet-vanne permet d'isoler la station de la conduite; il sert encore, en l'ouvrant partiellement, à opérer sous des chutes inférieures à 30 mètres, en créant des pertes de charge au passage du robinet. On peut ainsi opérer sur des modèles de dimensions réduites, tout en restant dans des proportions raisonnables, comme vitesse de rotation et comme débits. Mais cette fa-
L'installation est complétée par un canal de fuite, avec déversoir en mince paroi, du type de Bazin.

Mesure des puissances. - La mesure des puissances est effectuée au frein de Prony, le bras de levier du frein appuyant sur une bascule. La poulie du frein est refroidie intérieurement, et graissée extérieurement. Ce frein est calculé assez largement pour les puissances à mesurer, de sorte qu'il est d'une conduite facile, et les vitesses, mesurées directement en bout de l'arbre de la turbine, au moyen d'un tachymètre portatif, peuvent ètre assez facilement maintenues constantes pendant la durée d'un essai.

Mesure de la pression. - La pression, à l'axe de la bàche, est mesurée au moyen d'un manomètre à mercure, composé d'un tube en $\mathrm{U}$, dont l'une des branches communique avec la bâche, et dont l'autre branche débouche à l'air libre.
HIG. 1.

PLAN D.ENSEMBLE, de la Slation d Eludes de Riouperoux.
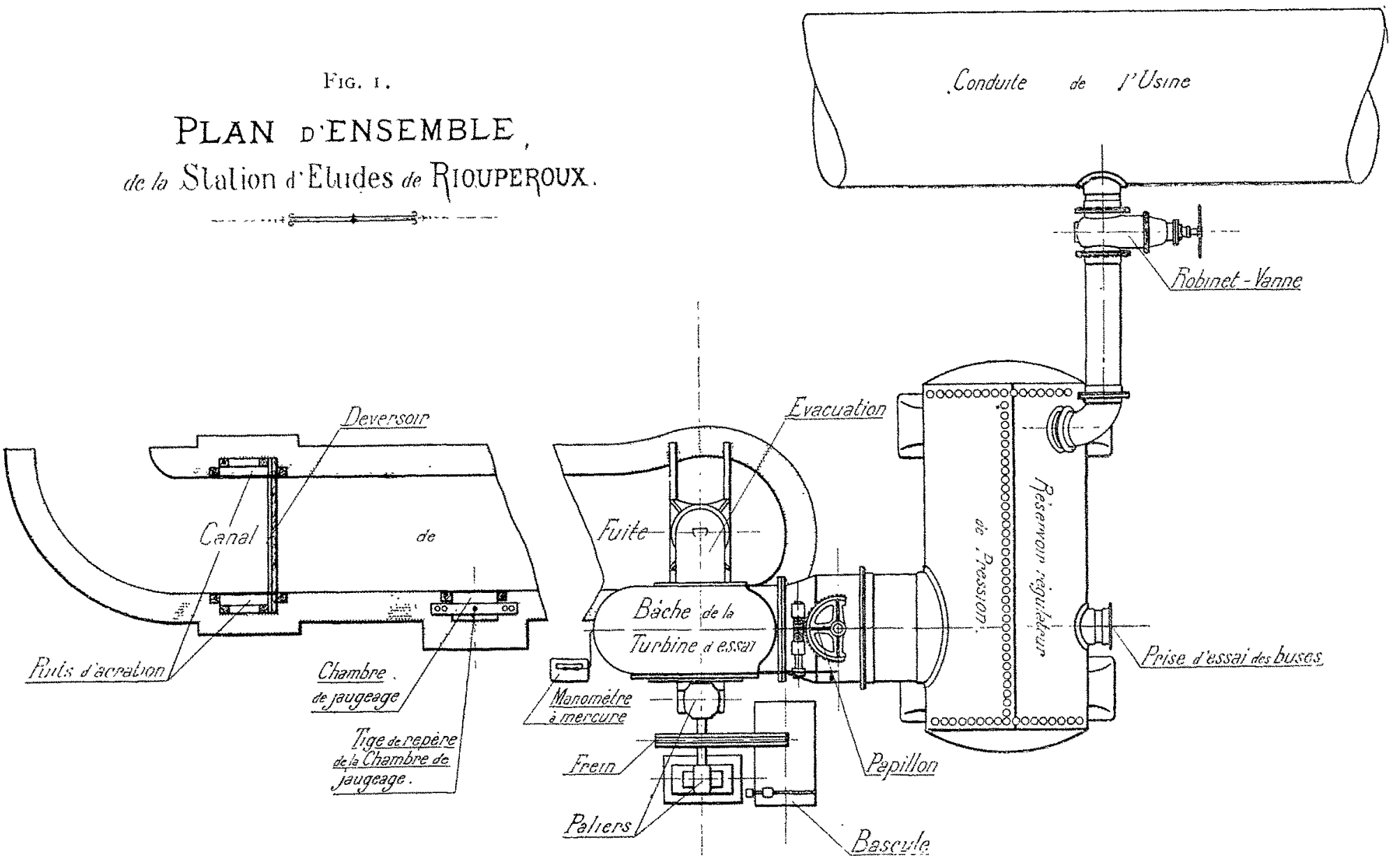

con de procéder pour obtenir une chute réduite présente l'inconvénient de créer des tourbillons après le robinet; on régularise alors l'écoulement en faisant arriver l'eau, non pas directement dans la bàche de la turbine d'essais, mais dans un réservoir en tôle d'acier d'assez grandes dimensions.

Sur ce réservoir régulateur sont branchées deux tubulures. L'une est destinée à porter des buses et injecteurs pour turbines à libre déviation. On laisse le jet s'écouler librement dans l'air, et on peut ainsi l'étudier sur une grande longueur, tant au point de vue de sa pureté de forme qu'au point de vue des pertes de charge que peut créer la buse.

L'autre tubulure porte un vannage à papillon, permettant d'isoler le réservoir régulateur de la bàche de la turbine d'essais, qui ne possède pas d'autre vannage.

La bàche est de forme spiraloüde, et, sur l'arbre horizontal, est calée une poulie de frein.
L'aspiration est mesurée également en colonne de mercure, ce qui permet l'étude des diffuseurs et tuyaux d'aspiration.

Mesure des débits. - La mesure des' débits s'effectue au moyen d'un déversoir en mince paroi, du type décrit par Bazin dans les Annales des Ponts-et-Chaussées de 1888 et I $\left.890 .{ }^{*}\right)$

Le déversoir, dont la crète est constituée par une tôle de $5 \mathrm{~mm}$. d'épaisseur taillée en biseau, tient toute la largeur du canal; il n'y a donc pas de contraction latérale, et l'aération de la lame déversante se fait au moyen de deux puits latéraux. Les parois du canal sont prolongées, au droit de ces puits, et au-dessus du déversoir, par des tôles.

La mesure de la hauteur de la lame d'eau au-dessus de la

(*) On en trouvera également la description dans la publication ctiée précédemment. 
crête du déversoir se fait dans une chambre latérale, fermée du côté du canal par une tôle, et communiquant avec lui seulement par deux trous percés à la partie inférieure. Ce procédé, employé ćgalement par Bazin, permet d'obtenir dans cette chambre de mesure une surface libre parfaitement calme, et de faire ainsi les lectures avec une très grande précision, au moyen d'une pointe verticale, portée par un tes courbes résultant des essais effectués avec une roue et un distributeur de turbine centripète à grande vitesse.

La bàche d'essais ne comportant pas de vannage, ces résultats correspondent au fonctionnement d'une turbine ì pleine ouverture.

Les essais ont été effectués en partant de l'arrêt, la turbine étant bloquée par le frein; une première série de lec-

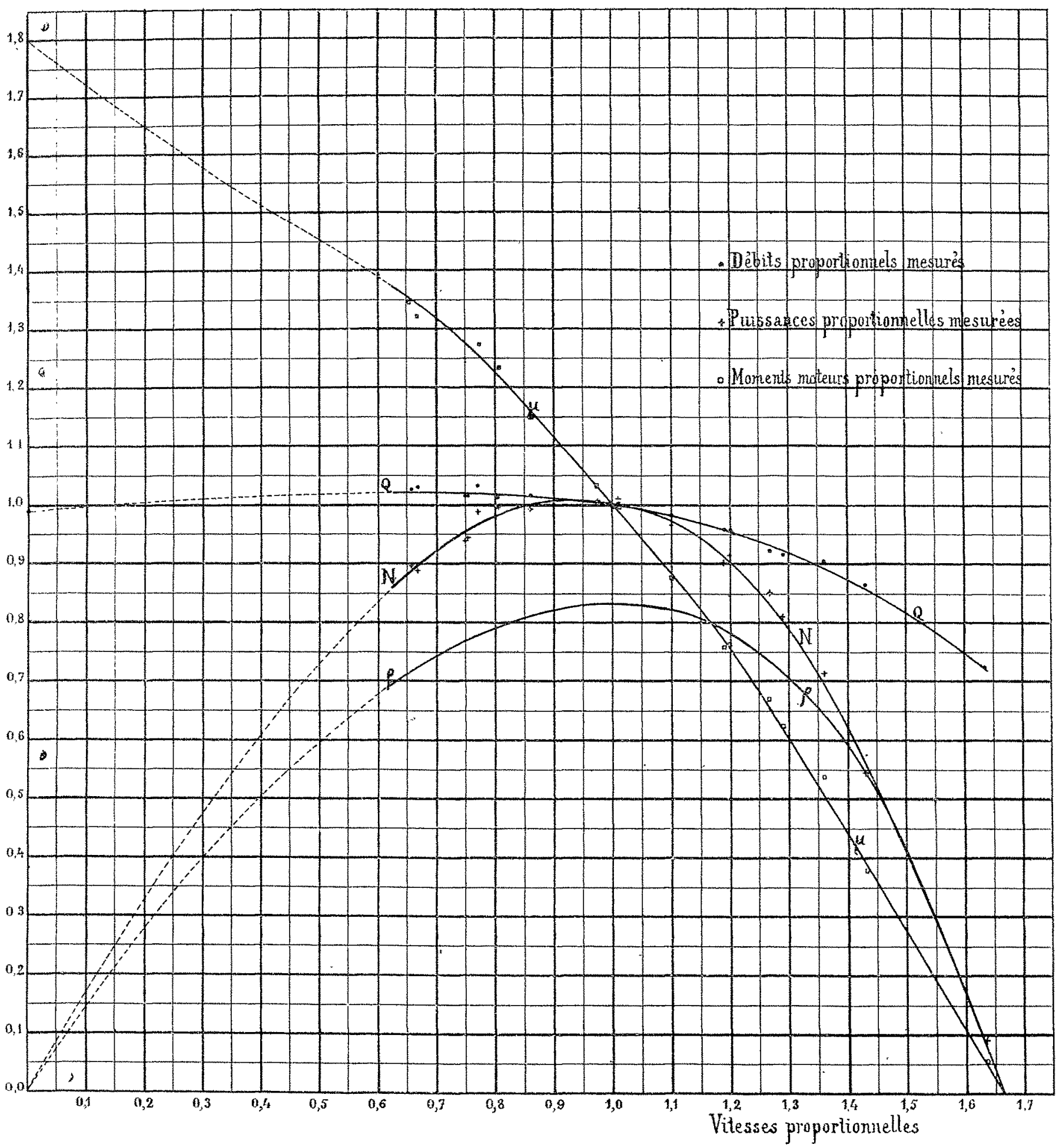

Fig. 2. - Courbes carnctéristiques de la turbine essayée.

batti fixe, et que l'on amene it affeurer la surface de l'eau dans le puits. Les conditions des expériences se rapprochant aussi parfaitement que possible de celles de Bazin, on peut, en employant les coefficients qu'il a déterminés, mesurer les débits avec une grande exactitude.

Exemple d'un essai effeclué dans la station. - Nous donnons ci-contre, figure 2 , à titre d'exemple, les différen. tures à vitesse croissante était faite en desserrant progressivement le frein jusqu'a l'emballement complet de la turbine; puis on effectuait une deuxième série de lectures à vitesse décroîssante, depuis l'emballement jusqu'à l'arrêt.

Pendant tous ces essais, on s'est efforcé de maintenir la pression aussi constante que possible, en agissant sur le robinet-vanne. 
Les différentes courbes représentent des nombres proportionnels aux débits, puissances, etc., en prenant pour unité les valeurs qui correspondent à la vitesse normale de la turbine. Lés vitesses proportionnelles sont portées en abcisses. Les parties ponctuées des courbes correspondent à des va. leur supposées entre les valeurs obtenues à l'arrêt et celles correspondant aux plus faibles vitesses réalisées.

Pour construire les courbes, on a transformé d'abord les différents résultats obtenus aux essais en opérant sous une chute sensiblement constante, de manière à les ramener tous à une chute rigoureusement constante, et cela de la facon suivante.

Soit $H$ la chute sous laquelle a eu lieu l'essai ; soient $Q$ le débit mesuré au déversoir, $n$ le nombre de tours mesuré sur larbre de la turbine, et $P$ la puissance mesurée au frein. Les rendements obtenus par la turbine fonctionnant sous lia chute $H$ seront les mêmes que sous la chute $H$, si on fait tourner la turbine à la vitesse

$$
n^{\prime}=n \sqrt{\frac{\bar{H}}{H}}
$$

Dans ces conditions, son débit et sa puissance seront donnés respectivement par:

$$
Q^{\prime}=Q \sqrt{\bar{H}} \quad P^{\prime}=P\left(\frac{H}{H}\right)^{\frac{3}{2}}
$$

et cela en vertu du principe de la similitude mécanique. Ceci suppose toutefois - et, comme nous l'avons signalé, c'est le cas dans les essais effectués ici - que $H^{\prime}$ est assez voisin de $H$ pour que les résistances passives ne varient pas sensiblement en passant d'une chute à l'autre.

La figure 2 comporte 4 courbes transformées.

$1^{\circ}$ Courbe des débils proportionnels (courbe Q). - Cette courbe présente une allure elliptique, avec les caractéristiques signalées par les différents auteurs, en particulier par le docteur Prasil, professeur au Polytechnikum de Zurich, à savoir: que le débit part d'une valeur déterminée à l'arrêt, puis croît avec la vitesse jusqu'à un certain maximum, pour décroître ensuite jusquà une valeur inférieure à celle correspondant ì l'arrêt.

Le maximum du débit correspond d'ailleurs à une vitesse inférieure à celle du maximun de rendement.

$2^{\circ}$ Courbe des moments moleurs proportionnels (Courbe p.). - L'allure général de cette courbe se rapproche de celle d'une droite; autrement dit, on peut admettre, d'une manière rapprochée, que le moment moteur est inversement proportionnel a la vitesse. En réalité, la courbe est d'abord convexe vers les 3 positifs, en partant de l'arrêt, puis elle devient concave pour les grandes vitesses.

$3^{\circ}$ Courbe des Puissances proportionnelles (Courbe N).La courbe a une allure générale parabolique; son maximum a lieu pour une vitesse inférieure à celle du maximum de rendement. Ce maximum partage la courbe en deux branches nettement dissymétriques; la puissance croît jusqu'au maximum plus lentement qu'elle ne décroît ensuite.

$4^{\circ}$ Courbe des rendements (Courbe p).- Cette courbc a la même allure parabolique que celle des puissances, mais son maximum (83 pour roo) a lieu pour la vitesse de régime de la turbine; on vérifie sur cette courbe que le rendement d'une turbine varie assez peu quand on fait varier sa vitesse de 5 pour 100 au-dessus et au-dessous de la vitesse de régime. Le maximum partage également la courbe en deux branches dissymétriques, le rendement diminuant plus vite pour les vitesses supérieures a la normale que pour les vitesses inférieures.

Plus de vingt séries d'essais ont été effectuées depuis six mois à la station de Rioupéroux, par les Ingénieurs du Service des Essais de la maison Neyret-Brenuer et Cie, tant sur des roues et distributeurs de turbines centripètes que sur des buses à aiguille et à bascule. Ces essais ont permis d'élucider beaucoup de points de détail, qu'il est difficile, sinon impossible, d'étudier dans les installations industrielles; la construction ne peut qu'y gagner, en précision d'abord, puisqu'on opère d'après des données vérifiées par l'expérience, en rapidité, ensuite, puisqu'on marche droit au but, sans tâtonnements.

Cette station d'études, due à une initiative privée, cst forcément un peu sommaire. Mais, pour qui connait, en matière d'industrie, l'abìne qui sépare un a projet» d'une "exécution», il nous a paru que la chose valait la peine d'être signalée.

C'est un encouragement à faire plus et mieux; aussi nous ne doutons que la générosité des industriels intéressés ne permette à M. BArbiLton d'adjoindre à son Institut Electrotechnique, après tant d'efforts, le laboratoire d'Essais $\mathrm{Hy}$ drauliques, " son rêve " depuis longtemps, comme il le dit dans la préface de l'excellent opuscule de M. Côte.

\section{G. Serviere,}

Ingénicur, Ancien álève de l'Ecole Polytechnique.

\section{INFLUENGES DU SOLEIL ET DE LA LUNE SUR L'ÉTAT PHYSIQUE DU GLOBE}

I

\section{Loi générale sur les relations des phénomènes solaires avee ceux de la physique din globe terrestre $\left(^{*}\right)$}

I. Les Rkgrons d'Activite du SolkH. - Dans de précédeules publications, jai ćlabli que oertains froupes de fucules du Soleil persistent parfois plusieurs annés a la surface de lastre; quan sein de ces groupes persistants des taches se forment, se dévelop. pent, disparaissent et soni remplacées, plus lard par d'autres.

Les facules, surlout les groupes tres persistants, paraissent itre ainsi le phénomène fondamontal de la photosphère, tandis que les taches sonk des phénomènes secondaires.

Les observations faites au Pic du Midi el it Bagnères depuis 1893, par mes collaboralems (MN. Latreille et Dort ou par moimeme) confiment, à ce point te vue, celles que javais faites autrefois a Lyon (1885-1802) sous la direction de mon mattre Charles Anaró ; ol M. Guillaume, rui a continué a Lyon depuis 1893 les observations du Soleil, a retrouvé eussi cette persistance prolongie de certains groupes faculaires.

On sait, d'antre part, quari-dessus de la photosphère du Soleil, il existe une sorte d'atmosphc̀re de vapeurs métalliques (chromosphère) dont les plus légèress (hydrogene en particulier') forment les prolubérances roses que les mólhodes spectrales de Janssen el de Lockyor permeltent de voir en toul temps et que les spectrohéliographes creés par Deslandres el par Hale (en 1893) permettonl de photographier.

Les protubérances peuvent ctre comparées i des ćruptions ; elles jaillissent aulour des taches, au-dessus des faculos qui entourent toujours celles-ci, ou au-dessus des groupes de facules ne contenant pas de taches. Les facules semblent ainsi etre des espaces rendus plus brillants par l'eruption meme qui s'y produit.

Je résume ces nolions daus l'́nonce suivant : ll existe constam. ment ì la surface du Soleil un certain nombre de régions dont

(') Communication présentéc le 5 Aoủ 1908 , au Congres do Clrmont

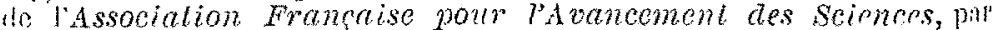

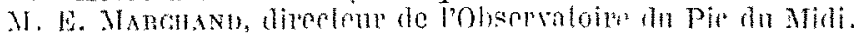

